CORRECTION

https://doi.org/10.1038/s41586-018-0111-5

\title{
Author Correction: Discovery of a selective catalytic p300/CBP inhibitor that targets lineage- specific tumours
}

Loren M. Lasko, Clarissa G. Jakob, Rohinton P. Edalji, Wei Qiu, Debra Montgomery, Enrico L. Digiammarino, T. Matt Hansen, Roberto M. Risi, Robin Frey, Vlasios Manaves, Bailin Shaw, Mikkel Algire, Paul Hessler, Lloyd T. Lam, Tamar Uziel, Emily Faivre, Debra Ferguson, Fritz G. Buchanan, Ruth L. Martin, Maricel Torrent, Gary G. Chiang, Kannan Karukurichi, J. William Langston, Brian T. Weinert, Chunaram Choudhary, Peter de Vries, Arthur F. Kluge, Michael A. Patane, John H. Van Drie, Ce Wang, David McElligott, Edward A. Kesicki, Ronen Marmorstein, Chaohong Sun, Philip A. Cole, Saul H. Rosenberg, Michael R. Michaelides, Albert Lai \& Kenneth D. Bromberg

Correction to: Nature https://doi.org/10.1038/nature24028, published online 27 September 2017.

In the originally published version of this Letter, the authors Arthur F. Kluge, Michael A. Patane and Ce Wang were inadvertently omitted from the author list. Their affiliations are: I-to-D, Inc., PO Box 6177, Lincoln, Massachusetts 01773, USA (A.F.K.); Mitobridge, Inc. 1030 Massachusetts Avenue, Cambridge, Massachusetts 02139, USA (M.A.P.); and China Novartis Institutes for BioMedical Research, No. 4218 Jinke Road, Zhangjiang Hi-Tech Park, Pudong District, Shanghai 201203, China (C.W.). These authors contributed to the interpretation of results and design of compounds. In addition, author 'Edward A. Kesicki' was misspelled as 'Ed Kesicki'. These errors have been corrected online.

In Fig. 1a (structure 4) of this Letter, the A-485 structure incorrectly included a sulfur atom (S) instead of a carbon (C) at the spiro centre. Figure 1 has been corrected online (see Supplementary Information to this Amendment for the original Fig. 1a, structure 4). In Fig. $4 \mathrm{~d}$ of this Letter, the units for enzalutamide (Enz) and A-485 were incorrectly listed as millimolar $(\mathrm{mM})$ instead of micromolar $(\mu \mathrm{M})$. Figure 4 has been corrected online. In Extended Data Fig. 7e of this Letter, the headings ' $-\mathrm{DHT}$ ' and ' $+\mathrm{DHT}$ ' were missing from the top of the western blots; this figure has been corrected online.

The third sentence of the main text should have read: "This led to two confirmed hits, a hydantoin (Fig. 1a, 1) and a conjugated thiazolidinedione (Fig. 1a, 2), which were similar to the previously described hit that inhibited Rtt $109^{8}$ and the hit $\mathrm{C} 375^{6}$, respectively", rather than "This led to two confirmed hits, a hydantoin (Fig. 1a, 1) and a conjugated thiazolidinedione (Fig. 1a, 2), which were similar to the previously described hits that inhibited Rtt $109^{8}$ and $\mathrm{C} 375^{6}$, respectively. This has been corrected online.

Finally, the following sentence has been added to the 'Competing interests' statement: A.F.K. and M.A.P were consultants to Acylin at the time of the study. C.W. was an employee of BioDuro, which was contracted by Acylin at the time of the study.

Supplementary Information is available in the online version of this Amendment. 